OPEN ACCESS

Edited by:

Xiaoshan Wu,

Central South University, China

Reviewed by:

Luyuan Jin,

Capital Medical University, China

Ruili Yang,

Peking University Hospital of Stomatology, China

${ }^{*}$ Correspondence:

Jinhua Yu

yujinhua@njmu.edu.cn

Specialty section: This article was submitted to Regenerative Dentistry, a section of the journal Frontiers in Dental Medicine

Received: 31 October 2021 Accepted: 31 December 2021 Published: 08 March 2022

Citation:

Yan C and Yu J (2022) Noncoding RNA in Extracellular Vesicles Regulate Differentiation of Mesenchymal Stem Cells. Front. Dent. Med. 2:806001.

doi: 10.3389/fdmed.2021.806001

\section{Noncoding RNA in Extracellular Vesicles Regulate Differentiation of Mesenchymal Stem Cells}

\author{
Chaoting Yan ${ }^{1,2,3}$ and Jinhua Yu ${ }^{1,2 *}$ \\ ${ }^{1}$ Key Laboratory of Oral Diseases of Jiangsu Province and Stomatological Institute of Nanjing Medical University, Nanjing, \\ China, ${ }^{2}$ Endodontic Department, School of Stomatology, Nanjing Medical University, Nanjing, China, ${ }^{3}$ Department of \\ Stomatology, The First People's Hospital of Lianyungang, Lianyungang Clinical Medical College of Nanjing Medical University, \\ Lianyungang, China
}

To achieve the desired outcome in tissue engineering regeneration, mesenchymal stem cells need to undergo a series of biological processes, including differentiating into the ideal target cells. The extracellular vesicle (EV) in the microenvironment contributes toward determining the fate of the cells with epigenetic regulation, particularly from noncoding RNA (ncRNA), and exerts transportation and protective effects on ncRNAs. We focused on the components and functions of ncRNA (particularly microRNA) in the EVs. The EVs modified by the ncRNA favor tissue regeneration and pose a potential challenge.

Keywords: noncoding RNA, extracellular vesicles, mesenchymal stem cell, exosome, differentiation

\section{INTRODUCTION}

Mesenchymal stem cell (MSC) therapy solves several problems related to the paracrine system, and it is not known for its salutary or rescue effects (1). Transplantation relies on the proliferation, self-renewal, and differentiation of the MSCs to achieve the regenerative goal, but it is limited by donator and guidelines. Cell-free therapy is also known as cell homing, which depends on the host cell under the given microenvironment to complete the task (2). The factors influencing the results of cell transplantation therapy also affect the results of cell-free therapy, including quality, reproducibility, and production potency (3). Extracellular vesicle (EV) from MSC (MSC$\mathrm{EV}$ ), especially highly homologous MSC, could perform tissue regeneration and immunoregulation function and help in avoiding the aforementioned problems (4). For example, bone marrow mesenchymal stem cell (BMSC) exosome rescues radiation-related bone defects, which is similar to MSC therapy (5). Exosome from dental pulp stem cells has been demonstrated to be a biomimetic tool for pulp regeneration (6). MSC-originated exosome has been shown to promote nerve regeneration in an animal model (7). The regulation of each step of regeneration by EVs is critical. Researchers have explored the bioactive content of EVs and have focused on the key aspect of noncoding RNA (ncRNA) (4). There seems to be some connection between EV and ncRNA. The parent cell targets the recipient cell via EV cargo, such as ncRNA, and leads to a biological process (8). Exosome from the MSC mainly inhibits immunity and attenuate injury by delivery miR-182 $(9,10)$. Release of exosome and microRNA (miRNA) is significant for BMSC function and bone homeostasis and can be inhibited by the simultaneous demethylation of P2rX7 (11). Shuttling cytosolic ncRNA, EVs perform epigenetic regulation as a special nano-communication system (12). The transcriptional and post-transcriptional genetic modification provides a controllable, manageable, and feasible avenue for regulating the differentiation of MSCs into lineage-specific 


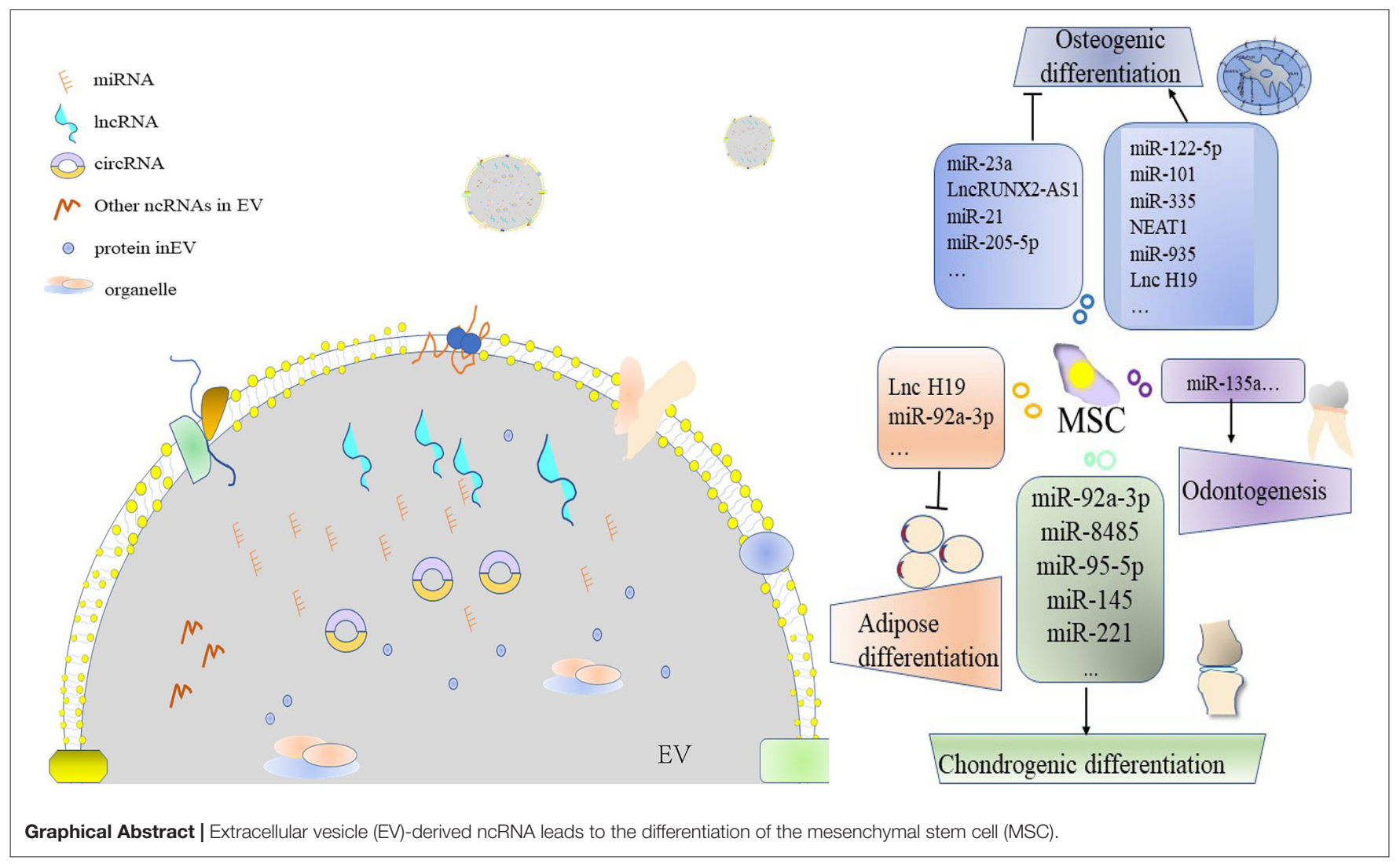

cells. The innate immunomodulatory activity of the MSCs is co-constructed by exosomal miRNA and the arresting domain-containing protein of the micro vesicles (13). The aforementioned crosstalk is useful for cell-free MSC therapy.

Extracellular vesicles (EVs) can be classified into three types according to their biogenesis and partial-overlap size: exosome $[30-100 \mathrm{~nm}$ (14) or 30-130 nm], microvesicles $(100-1,000 \mathrm{~nm})$, and apoptotic bodies (50-4,000 nm) (4). Additionally, microvesicles are also called large size EVs (lEVs) or microparticles (MPs), and they were believed to carry cell organelles and bioactive molecules, such as ncRNA (15). According to the originating cells, EVs can be classified into tumor-derived extracellular (TEVs), outer membrane vesicles (OMVs) from bacteria, plant extracellular vesicles, and so on (1618); furthermore, the exosomes can be distinguished into CD63, CD9, CD81, and cavelion1 subpopulations according to their immunological features of the membrane (19). The membrane of the EVs protects the RNAs from low PH (20) and helps them to resist nuclease activity (21). Because of the protective effect, low immunogenicity, and stability of the membrane, EVs serves as a potential carrier for nano-delivery treatments (22). The EVs mainly contain protein, nucleic acid, and lipid and vary based on origin and condition (12). miRNAs are prone to enrich in exosomes comparing to other EV species (23). Furthermore, the nucleotide sequence motif is the ticket for miRNA packaging into exosomes of specific types of cells (24). The plasma concentration of individual RNAs is another decisive factor (20). Blebbing from the dying cells, the apoptotic body mainly carries tightly-packed organelles, proteins, and mRNAs $(25,26)$. The apoptotic bodies contain miRNA, and both can activate the immune response and promote cell death (27). Along with other factors, EVs regulate the biological behavior of the target cell, such as differentiation (28). The exosome from MSC has a considerable role in oral-facial bone regeneration (29). This article summarizes the ncRNA cargo in the EV, the manner of ncRNA packaging, and the concerted effects of EV and ncRNA on the target cell.

\section{ncRNA EXISTS IN EVS}

\section{Interest in This Field}

There are several publications on EVs and ncRNA, especially exosome and miRNA, and they are increasing exponentially. For example, in 2020 alone, articles pertaining to the mentioned keywords amount to 1,390. Hence, over the past years, the topic has received immense attention, and researchers have contributed to the development of exosome-based ncRNA delivery and the biological behaviors of the recipient cells. It was first reported in 2007 that "exosomal" regulation of cell reprogramming is dependent on miRNA and mRNA (30). The genetic information of the EVs resembles that of the parent cell (12), and miRNA always serves as the biologically active material in the EV for downstream clarification (31). 


\section{The Achievement in This Field}

Many types of ncRNAs, including transfer RNA (tRNA), Y RNA, ribosomal RNA (rRNA), PIWI-interacting RNA (piRNA), circular RNA (circRNA), and unannotated RNAs are contained in the exosomes $(4,32-34)$. It has been demonstrated that RNase I generates extracellular Y RNA and tRNA (21), and that these RNAs partially exist in the open extracellular space (35). A stressregulated vesicle as tiRNA (tRNA) signaling approach promotes proliferation, protein translation, and differentiation (36). PIWIpiRNA complexes silence the targets during transcription via transporting by MSC-EVs $(37,38)$. The release of EVs is believed to be the clearing mechanism of circRNA from the plasma (39), while EV also protects circRNA to contain the equilibrium (40). Long noncoding RNAs (lncRNAs) from exosomes could be a biomarker for disease (41), and the IncRNA-miRNA-mRNA network has been investigated in exosomes (42). Furthermore, the network function can be described as follows: (1) function as a sponge; (2) co-expression of ncRNAs; (3) reciprocal repression; (4) role of miRNAs as negative regulators of lncRNAs (43); (5) other unconventional miRNA functions (44). Despite the continued emergence of new species, nowadays, most reports are focused on miRNA and lncRNA and great progress has been made (45).

EVs have the potential to aid in the diagnosis, research, and application because of the packaging of the cytoplasmic contents into natural lipid membranes and their shuttle through the natural barrier (46). Remarkably, miRNA selectively captured in the exosomes is partially dependent on the endosomal sorting complex required for transport (ESCRT) $(47,48)$, which is the classical exosome biogenesis mechanism. Furthermore, other factors are also involved in miRNA packaging. These include CD63, MHC1, CD47 (49), neutral sphingomyelinase 2 (nSMase2)-ceramide (47), miRNA associated protein of RNAinduced silencing complex (RISC) component (50), RNA binding protein(synergistical function), membrane transportation (51), and motif-binding protein of RNA binding protein $(52,53)$. The isolation manner also impacts the RNA cargoes via lipoproteins (54). The ncRNA content of the EV is also determined by the cytoplasmic level of the genes of the parent cell (12).

Naturally occurring exosomes can be transported to neighboring and remote sites via body fluids (55) and alter multiple genes and signaling pathways in the target cells (56). The exosomes modified by tissue engineering navigate the targets by membrane protein modification (57) or the physics method (58).

The miRNA expression undergoes striking changes based on the cell-cell communication in a specific microenvironment (59). This interaction is also named "exosome-shuttle miRNAs" (60). Remarkably, interaction also exists between the extracellular matrix and the exosome (61).

\section{Methodology in This Field}

Using the advanced methods of quantitative real-time polymerase chain reaction, microarray, and sequencing technology, genomic complexities and functions were gradually revealed by biological experiments or bioinformatic analysis.
Databases, such as Exocarta, Vesiclepedia, and EVpedia, make the above information publicly accessible and develop the relevant software, thereby creating a convenient platform for upstream and downstream investigations (62). Normally, the comparison has always been set among the EVs of different origins because EVs and their originating cells always play similar roles in biological behavior. However, differential expression also exists between the originating cells and their exosomes $(63,64)$.

\section{ORIGINATING CELLS CONTRIBUTE TO EV-DERIVED ncRNAS}

In the early stages of life, the epigenome has been determined by both inherited and nutritional factors. Subsequently, the environmental factors become involved, that is, external stimuli (65). Cells present in the microenvironment experience various physical, chemical, and biological changes, and they can adjust themselves based on epigenetic regulation and delivery crosstalk information, such as the release of content-reprogramed EVs (66-68). Generally, MSCs from craniofacial tissues are roughly divided into BMSC, dental MSC, and adipose stem cell (ADSC) with potential for hard and soft tissue repair and regeneration. Under specific conditions (origin, inflammation, aging and apoptosis, physical stimulus, ncRNA modification, and differentiation), the cells differ in their ability to express EVs (Table 1).

\section{The Expression of ncRNA in MSC-Origin EVs}

Exosomes from ADSC promote neuronal survival by transporting MALAT1 (69). Microvesicles from the same parent cell promote both proliferation and migration by delivering miR-210 (70). ADSC and BMSC share highly identical small RNA profiles (mainly involving miRNA and snoRNA), and so does the exosome. However, differential expression of tRNA was observed, which could be explained by tissue origin and stemness (33). The expression of piRNA in exosomes from stem cells from apical papilla (SCAP) provides a novel insight into the functions, such as biological regulation (38). miR-1470 is one of the exosomal biomarkers of BMSCs for its high expression when exosomes from fibroblast serve as the control (71). Equipped with miR-92a-3p, miR-26a-5p, miR-23a-3p, miR-103a-3p, and miR-181a-5p, exosome from neuronal stem cell has therapeutic potential (78).

\section{The Modification of Cells Triggers EV to Transfer ncRNAs}

Macrophage polarization changes the EV function in bone repair/regeneration by adjusting enclosed miRNA (79). Inflammation modifies the loading and quality of EVs by RNA binding protein FMR1 and ESCRT pathway (80).

Aging leads to the change of exosome from BMSC, which clarifies immune-associated miRNA profiles, and toll-like receptor 4-regulating miR-21-5p is highly expressed in the prepubertal group (81). Similarly, the upregulation of miR-128-3p 
TABLE 1 | A summary of several common ncRNA derived from extracellular vesicles (EVs) of mesenchymal stem cells (MSCs).

\begin{tabular}{|c|c|c|c|}
\hline $\begin{array}{l}\text { Noncoding RNA- target } \\
\text { gene }\end{array}$ & Target cell & Parent cell and EV type & Biological behavior \\
\hline MALAT1- PKC\&II (69) & HT22 neuronal cells & ADSC and exosome & Survival and proliferation \\
\hline miR-210-RUNX3 (70) & human umbilical vein endothelial cells & ADSC and microvesicle & Proliferation, migration and invasion \\
\hline miR-1470-c-Jun (71) & CD4 + CD25 + FOXP3 + Tregs & BMSC and exosome & Differentiation \\
\hline miR-128-3p-Smad5 (72) & MSC & aged MSC and exosome & Inhibition of osteogenic differentiation \\
\hline miR-31a-5p-SATB2 (73) & BMSC & BMSC from aged rats and exosome & Osteoclastogenesis \\
\hline miR-126-SPRED1 (74) & human umbilical vein endothelial cell & $\begin{array}{l}\text { hypoxic human umbilical cord MSC and } \\
\text { exosome }\end{array}$ & Angiogenesis, proliferation and migration \\
\hline miR-181c-TLR4 (75) & macrophages & human umbilical cord MSC and exosome & Inflammation reverse \\
\hline H19-miR-106-Angpt1 (76) & $/^{*}$ & BMSC and exosome & Osteogenesis and angiogenesis \\
\hline $\begin{array}{l}\text { miR-122-5p, miR-25-3p, } \\
\text { and miR-142-5p } \Delta(77)\end{array}$ & rat BMSCs & $\begin{array}{l}\text { Osteogenic differentiated human } \\
\text { periodontal ligament stem cell and } \\
\text { exosomes }\end{array}$ & Osteogenic differentiation \\
\hline
\end{tabular}

*This conclusion is derived from experiment in vivo.

$\Delta$ This article does not involve downstream functional verification

in the exosome of the BMSC of an aged mouse inhibits fracture healing (72). miR-31a-5p from BMSC exosome of aged rats promotes osteoclastic and attenuates osteoblastic differentiation, which is similar behaviors when compared with its origin (73). Staurosporine-treatment results in ribosomal RNA cleavage and degradation (82).

Hypoxia preconditioning changes the content of miR-126 in the exosomes from MSCs and makes it fit for bone fracture healing (74). Hypoxia also induces angiogenic miR$135 \mathrm{~b}$ to increase expression in exosomes (83). Mechanical strain upregulates miR-181b-5p in the exosomes of osteocytes (84).

miRNA-modified originating cell leads to the same miRNA increase in the exosome. In other words, transfection of miRNA can promote the secretion of itself in the supernatant $(4,85)$. For example, miR-181-5p modified adipose stem cell delivers therapeutic EV to the damaged site and contributes to recovery via activating autography (86). miR-181c-overexpressed MSC exosome significantly reduces the burn origin inflammation (75). Exogenous mimic or inhibitor has been reported to regulate the downstream cell with more efficiency and less toxicity compared with the conventional manner (87). Microvesicles from miR34a overexpressing BMSC carry three-fold higher miR-34a than the control (88). Transfection of miR-20a makes BMSC-EV favorable for alveolar bone-implant osteointegration via proosteogenic effects (89). Similarly, osteogenic differentiationrelated to miR-375 can be capsulated by adipose stem cell exosome and the exosome can induce BMSC osteogenic commit (90).

During osteogenesis, BMSC releases lncH19-containing exosomes which have been validated by observing the bone microstructure of immunocompromised nude mice (76). Osteogenic induction modifies the EV cargoes of PDLSC with miR-122-5p as the top one by miRNA differential expression analysis (77). Moreover, different cell types possess distinct RNA profiles (91), and the miRNA in adipose-derived exosomes is related to insulin-associated metabolism (92).

\section{EV-DERIVED ncRNA INDUCES DIFFERENTIATION}

The biogenesis of EVs is implicated in a series of biological processes, including the differentiation of MSCs. Exchange of EV ncRNA cargo is also important in achieving cell population homeostasis between the progenitor cell and the differentiated cell (93). RNase completely abolishes the biological effect of exosome-like microvesicles, which indicates the participation of RNA-like components $(94,95)$. EV reprograms MSCs and makes them produce more specific EVs to disseminate miRNA and mRNA (96).

\section{EV-Derived ncRNA Induces Osteogenic Differentiation}

Exosomes carrying miR-122-5p negatively regulate SPRY2 to initiate osteoblastic phenotypes (97). Radiation-induced gingival fibroblasts highly express miR-23a in their exosomes, thereby resulting in the osteogenic differentiation of BMSC (98). The lncRNA RUNX2-AS inhibits the key osteogenic regulator-, RNUX2, by the transportation of exosomes (99). miR-21/Smad7 axis detected by the exosome of BMSC is the mechanism of negative osteogenesis in osteoporosis patients (100). After 21 days of osteogenic inducement, miR-101 is upregulated in BMSC-derived exosomes, and the target demonstrates osteogenic potential upon functional verification (101). $\mathrm{miR}-335 / \mathrm{VapB} / \mathrm{Wnt} / \beta$-catenin axis promotes bone fracture recovery and osteoblast differentiation, which is attributed to BMSC-derived EVs (102). Osteogenic and odontogenic lineage progression includes the epigenic involvement of RUNX2 and BMP2 (103, 104). Furthermore, exosomal miR-135a enhances the output of the dentin matrix via $\mathrm{Wnt} / \beta$-catenin signaling pathway and contributes to tooth development (105). NEAT1containing exosomes induce BMSC osteogenic commitment by sponging miR-205-5p (106). MiR-223 decreased exosomes promote differentiation into osteoblasts (107). Exosomes from 
BMSC promote osteoblast proliferation and differentiation by releasing STAT1-targeting miR-935 (108).

\section{EV-Derived ncRNA Induces Chondrogenic Differentiation}

Exosomal miR-92a-3p regulates chondrogenic development by directly targeting Wnt5A and therefore holds therapeutic potential in osteoarthritis (109). Chondrogenic differentiation also makes the BMSC exosomes highly express 35 kinds of miRNA, including miR-92a (110). Chondrocytes induce BMSC chondrogenic differentiation via the delivery of miR-8485containing exosomes in a co-culture system (111). miR-95-5p is overexpressed in the chondrocytes, and the chondrocytes in turn produce chondrogenic exosomes, which can inhibit HDAC2/8 (112). Adipose stem cells deliver exosomal miR-145 and miR-221 to regenerate the cartilage and ameliorate osteoarthritis (113).

\section{EV-Derived ncRNA Induces Angiogenic Differentiation}

Vascularization is considered the key factor for successful transplantation and regeneration as vascular supply necessary nutrition and oxygen. Stem cells from deciduous teeth (SHED) aggregates secrete miR-26-enriched exosomes to forge a suitable microenvironment for pulp regeneration (114). Serum exosomal miR-1956 stimulates the angiogenic differentiation of adipose stem cells by activating Notch-1 (115).

\section{EV-Derived ncRNA Induces Neurogenic Differentiation}

miR-17-92 cluster-enriched MSC-exosomes augment neural plasticity and functional recovery by regulating the cluster target gene (116). Transferring exosomal miR-133b and miR-124-3p regulates nerve outgrowth and neurological disease recovery and reduces neuroinflammation $(117,118)$. miR-126-loaded MSC exosomes may be a candidate for the treatment of neurological injury, with excellent angiogenic and neurogenic potentials (119). MSC exosomal miR-199a-3p/145-5p plays a critical role in neuronal differentiation (120).

\section{EV-Derived ncRNA Induces Adipogenic Differentiation}

Exosomal miR-92a-3p regulates $\mathrm{C} / \mathrm{EBP} \alpha$ at the posttranscriptional level to block adipogenic differentiation of

\section{REFERENCES}

1. Bernardo ME, Pagliara D, and Locatelli F. Mesenchymal stromal cell therapy: a revolution in regenerative medicine? Bone Marrow Transplant. (2012) 47:164-71. doi: 10.1038/bmt.2011.81

2. Eramo S, Orcid Id, Natali A, Pinna R, and Milia E. Dental pulp regeneration via cell homing. Int Endod J. (2018) 51:405-19. doi: 10.1111/iej.12868

3. Phinney DG, and Pittenger MF. Concise review: MSC-derived exosomes for cell-free therapy. Stem Cells. (2017) 35:851-8. doi: 10.1002/stem.2575

4. Qiu G, Zheng G, Ge M, Wang J, Huang R, Shu Q, et al. Mesenchymal stem cell-derived extracellular vesicles affect disease outcomes via endorsement of macrophage polarization. Stem Cell Res Ther. (2018) 9:320. doi: 10.1186/s13287-018-1069-9 adipose stem cells (121). Obesity and high-fat treatment break the bone-fat equilibrium and downregulate the expression of lnc $\mathrm{H} 19$ in the circulation and output of BMSC-origin exosomes (122).

\section{CHALLENGES AND PROSPECTS}

Although organelles, cytokines, and lipids enrich EV constituents (123), this review implicates ncRNA. To facilitate clinical translation, high purity, low cost, and largescale exosome isolation techniques need to be developed (124). 3D culture and isolation by tangential flow filtration can potentially pave the road from bench to bed (125). A specific aptamer for exosomal application is also necessary since the exosomes significantly enhance the osteoblastic differentiation of BMSC in vitro but not in vivo (126).

Largescale studies on RNA according to the miRNA nomenclature guidelines are also inadequate; so are the studies on clusters and other EV-derived ncRNAs (excluding lncRNA and miRNA). Moreover, the autocrine system is an issue worth exploring (127).

It is hoped that EVs would be a better drug vehicle than lipids since they can pass natural biological barriers $(128,129)$ or exert functions with their anatomical apposition (130). They are safe for use in the field of oncology and can be modified using multiple methods (131). Moreover, "EV encapsulated RNA drugs" combined with engineering modification is a powerful cell-free gene therapy tool that holds considerable promise for regenerative therapy in the future.

\section{AUTHOR CONTRIBUTIONS}

CY contributed to the design and conception, manuscript writing, and final approval of the manuscript. JY contributed to the design and conception, manuscript revising, financial support, and final approval of the manuscript. Both authors have read and approved the final version of the manuscript.

\section{FUNDING}

This work was supported by the National Natural Science Foundation of China (grant numbers: 81873707 and 82170940).
5. Zuo R, Liu M, Wang Y, Li J, Wang W, Wu J, et al. BM-MSCderived exosomes alleviate radiation-induced bone loss by restoring the function of recipient BM-MSCs and activating Wnt/ $\beta$-catenin signaling. Stem Cell Res Ther. (2019) 10:30. doi: 10.1186/s13287-0181121-9

6. Huang CC, Narayanan R, Alapati S, Ravindran S. Exosomes as biomimetic tools for stem cell differentiation: applications in dental pulp tissue regeneration. Biomaterials. (2016) 111:103-15. doi: 10.1016/j.biomaterials.2016.09.029

7. Ma Y, Dong L, Zhou D, Li L, Zhang W, Zhen Y, et al. Extracellular vesicles from human umbilical cord mesenchymal stem cells improve nerve regeneration after sciatic nerve transection in rats. J Cell Mol Med. (2019) 23:2822-35. doi: 10.1111/jcmm.14190 
8. Rahmani A, Saleki K, Javanmehr N, Khodaparast J, Saadat P, and Nouri HR. Mesenchymal stem cell-derived extracellular vesicle-based therapies protect against coupled degeneration of the central nervous and vascular systems in stroke. Ageing Res Rev. (2020) 62:101106. doi: 10.1016/j.arr.2020.101106

9. Zhao J, Li X, Hu J, Chen F, Qiao S, Sun X, et al. Mesenchymal stromal cell-derived exosomes attenuate myocardial ischaemia-reperfusion injury through miR-182-regulated macrophage polarization. Cardiovasc Res. (2019) 115:1205-16. doi: 10.1093/cvr/cvz040

10. Burrello J, Monticone S, Gai C, Gomez Y, Kholia S, and Camussi G. Stem cell-derived extracellular vesicles and immune-modulation. Front Cell Dev Biol. (2016) 4:83. doi: 10.3389/fcell.2016.00083

11. Yang R, Yu T, Kou X, Gao X, Chen C, Liu D, et al. Tet1 and Tet2 maintain mesenchymal stem cell homeostasis via demethylation of the P2rX7 promoter. Nat Commun. (2018) 9:2143. doi: 10.1038/s41467-018-04464-6

12. Abels ER, and Breakefield XO. Introduction to extracellular vesicles: biogenesis, RNA cargo selection, content, release, and uptake Cell Mol Neurobiol. (2016) 36:301-12. doi: 10.1007/s10571-016-0366-Z

13. Phinney DG, Di Giuseppe M, Njah J, Sala E, Shiva S, St Croix CM, et al. Mesenchymal stem cells use extracellular vesicles to outsource mitophagy and shuttle microRNAs. Nat Commun. (2015) 6:8472. doi: $10.1038 /$ ncomms 9472

14. Shao L, Orcid Id, Zhang Y, Lan B, Wang J, Zhang Z, et al. MiRNAsequence indicates that mesenchymal stem cells and exosomes have similar mechanism to enhance cardiac repair. Biomed Res Int. (2017) 2017:4150705. doi: $10.1155 / 2017 / 4150705$

15. Osman A, Orcid Id, Benameur T, Korashy HM, Orcid Id, Zeidan A, et al. Interplay between endoplasmic reticulum stress and large extracellular vesicles (microparticles) in endothelial cell dysfunction. Biomedicines. (2020) 8:409. doi: 10.3390/biomedicines8100409

16. Wu CH, Silvers CR, Messing EM, and Lee YF. Bladder cancer extracellular vesicles drive tumorigenesis by inducing the unfolded protein response in endoplasmic reticulum of nonmalignant cells. J Biol Chem. (2019) 294:320718. doi: $10.1074 /$ jbc.RA118.006682

17. Jang SC, Kim SR, Yoon YJ, Park KS, Kim JH, Lee J, et al. In vivo kinetic biodistribution of nano-sized outer membrane vesicles derived from bacteria. Small. (2015) 11:456-61. doi: 10.1002/smll.201401803

18. Cai Q, Orcid Id, Qiao L, Wang M, He B, Lin FM, et al. Plants send small RNAs in extracellular vesicles to fungal pathogen to silence virulence genes. Science. (2018) 360:1126-9. doi: 10.1126/science.aar4142

19. Choi DY, Park JN, Paek SH, Choi SC, and Paek SH. Detecting earlystage malignant melanoma using a calcium switch-enriched exosome subpopulation containing tumor markers as a sample. Biosens Bioelectron. (2021) 198:113828 doi: 10.1016/j.bios.2021.113828

20. Izumi $\mathrm{H}$, Kosaka $\mathrm{N}$, Shimizu $\mathrm{T}$, Sekine $\mathrm{K}$, Ochiya $\mathrm{T}$, and Takase M. Bovine milk contains microRNA and messenger RNA that are stable under degradative conditions. J Dairy Sci. (2012) 95:4831-41. doi: 10.3168/jds.2012-5489

21. Nechooshtan G, Yunusov D, Chang K, and Gingeras TR. Processing by RNase 1 forms tRNA halves and distinct Y RNA fragments in the extracellular environment. Nucleic Acids Res. (2020) 48:8035-49. doi: 10.1093/nar/gkaa526

22. Burgess DJ. Signalling: vesicle vehicles of genetic information. Nat Rev Genet. (2014) 15:514. doi: 10.1038/nrg3780

23. Wei Z, Orcid Id, Batagov AO, Schinelli S, Wang J, Wang Y, et al. Coding and noncoding landscape of extracellular RNA released by human glioma stem cells. Nat Commun. (2017) 8:1145. doi: 10.1038/s41467-017-01196-x

24. Garcia-Martin R, Wang G, Brandao BB, Zanotto TM, Shah S, Kumar S, et al. MicroRNA sequence codes for small extracellular vesicle release and cellular. Nature. (2022) 601:446-51. doi: 10.1038/s41586-021-04234-3

25. Taylor RC, Cullen SP, and Martin SJ. Apoptosis: controlled demolition at the cellular level. Nat Rev Mol Cell Biol. (2008) 9:231-41. doi: 10.1038/nrm2312

26. Crescitelli R, Lässer C, Szabó TG, Kittel A, Eldh M, Dianzani I, et al. Distinct RNA profiles in subpopulations of extracellular vesicles: apoptotic bodies, microvesicles and exosomes. J Extracell Vesicles. (2013) 2:20677. doi: $10.3402 /$ jev.v2i0.20677

27. Reich CF III, Pisetsky DS. The content of DNA and RNA in microparticles released by Jurkat and HL-60 cells undergoing in vitro apoptosis. Exp Cell Res. (2009) 315:760-8. doi: 10.1016/j.yexcr.2008.12.014
28. Ponzetti M, Rucci N. Osteoblast differentiation and signaling: established concepts and emerging topics. Int J Mol Sci. (2021) 22:6651. doi: $10.3390 /$ ijms 22136651

29. Lee YH, Orcid Id, Park HK, Auh QS, Nah H, Lee JS, et al. Emerging potential of exosomes in regenerative medicine for temporomandibular joint osteoarthritis. Int J Mol Sci. (2020) 21:1541. doi: 10.3390/ijms21041541

30. Valadi H, Ekström K, Bossios A, Sjöstrand M, Lee JJ, Lötvall JO. Exosome-mediated transfer of mRNAs and microRNAs is a novel mechanism of genetic exchange between cells. Nat Cell Biol. (2007) 9:654-9. doi: 10.1038/ncb1596

31. Neviani P, Fabbri M. Exosomic microRNAs in the tumor microenvironment. Front Med. (2015) 2:47. doi: 10.3389/fmed.2015.00047

32. Li L, Li C, Wang S, Wang Z, Jiang J, Wang W, et al. Exosomes derived from hypoxic oral squamous cell carcinoma cells deliver miR-21 to normoxic cells to elicit a prometastatic phenotype. Cancer Res. (2016) 76:1770-80. doi: 10.1158/0008-5472.CAN-15-1625

33. Baglio SR, Rooijers K, Koppers-Lalic D, Verweij FJ, Pérez Lanzón M, Zini $\mathrm{N}$, et al. Human bone marrow- and adipose-mesenchymal stem cells secrete exosomes enriched in distinctive miRNA and tRNA species. Stem Cell Res Ther. (2015) 6:127. doi: 10.1186/s13287-015-0116-Z

34. Li Y, Zheng Q, Bao C, Li S, Guo W, Zhao J, et al. Circular RNA is enriched and stable in exosomes: a promising biomarker for cancer diagnosis. Cell Res. (2015) 25:981-4. doi: 10.1038/cr.2015.82

35. Tosar JP, Segovia M, Castellano M, Gámbaro F, Akiyama Y, Fagúndez P, et al. Fragmentation of extracellular ribosomes and tRNAs shapes the extracellular RNAome. Nucleic Acids Res. (2020) 48:12874-88. doi: 10.1093/nar/gk aa674

36. Kfoury YS, Ji F, Mazzola M, Sykes DB, Scherer AK, Anselmo A, et al. tiRNA signaling via stress-regulated vesicle transfer in the hematopoietic niche. Cell Stem Cell. (2021) 28:2090-103.e9. doi: 10.1016/j.stem.2021. 08.014

37. Czech B, Munafò M, Ciabrelli F, Eastwood EL, Fabry MH, Kneuss E, et al. piRNA-guided genome defense: from biogenesis to silencing. Annu Rev Genet. (2018) 52:131-57. doi: 10.1146/annurev-genet-120417-0 31441

38. Wang A, Liu J, Zhuang X, Yu S, Zhu S, Liu Y, et al. Identification and comparison of piRNA expression profiles of exosomes derived from human stem cells from the apical papilla and bone marrow mesenchymal stem cells. Stem Cells Dev. (2020) 29:511-20. doi: 10.1089/scd.2019.0277

39. Shi Z, Wang K, Xing Y, Yang X. CircNRIP1 encapsulated by bone marrow mesenchymal stem cell-derived extracellular vesicles aggravates osteosarcoma by modulating the miR-532-3p/AKT3/PI3K/AKT axis. Front Oncol. (2021) 11:658139. doi: 10.3389/fonc.2021.658139

40. Wang Y, Liu J, Ma J, Sun T, Zhou Q, Wang W, et al. Exosomal circRNAs: biogenesis, effect and application in human diseases. Mol Cancer. (2019) 18:116. doi: 10.1186/s12943-019-1041-z

41. Huang X, Sun L, Wen S, Deng D, Wan F, He X, et al. RNA sequencing of plasma exosomes revealed novel functional long noncoding RNAs in hepatocellular carcinoma. Cancer Sci. (2020) 111:3338-49 doi: $10.1111 /$ cas. 14516

42. Wu J, Orcid Id, Huang H, Huang W, Wang L, Xia X, et al. Analysis of exosomal lncRNA, miRNA and mRNA expression profiles and ceRNA network construction in endometriosis. Epigenomics. (2020) 12:1193-213. doi: 10.2217/epi-2020-0084

43. Huang Y. The novel regulatory role of IncRNA-miRNA-mRNA axis in cardiovascular diseases. J Cell Mol Med. (2018) 22:5768-75. doi: $10.1111 /$ jcmm. 13866

44. Dragomir MP, Knutsen E, Calin GA. SnapShot: unconventional miRNA functions. Cell. (2018) 174:1038-8.e1 doi: 10.1016/j.cell.2018.07.040

45. Li Q, Liu Q. Noncoding RNAs in cancer immunology. In: The Long and Short Non-coding RNAs in Cancer Biology, Song E, editor. Singapore: Springer Singapore (2016). p. 243-64. doi: 10.1007/978-981-10-1498-7_9

46. Jeyaram A, Jay SM. Preservation and storage stability of extracellular vesicles for therapeutic applications. AAPS J. (2017) 20:1. doi: 10.1208/s12248-017-0160-y

47. Kosaka N, Iguchi H, Yoshioka Y, Takeshita F, Matsuki Y, Ochiya T. Secretory mechanisms and intercellular transfer of microRNAs in living cells. J Biol Chem. (2010) 285:17442-52. doi: 10.1074/jbc.M110.107821 
48. Iavello A, Frech VS, Gai C, Deregibus MC, Quesenberry PJ, Camussi G. Role of Alix in miRNA packaging during extracellular vesicle biogenesis. Int J Mol Med. (2016) 37:958-66. doi: 10.3892/ijmm.2016.2488

49. Kaur S, Elkahloun AG, Arakelyan A, Young L, Myers TG, OtaizoCarrasquero F, et al. CD63, MHC class 1, and CD47 identify subsets of extracellular vesicles containing distinct populations of noncoding RNAs. Sci Rep. (2018) 8:2577. doi: 10.1038/s41598-018-20936-7

50. McKenzie AJ, Hoshino D, Hong NH, Cha DJ, Franklin JL, Coffey RJ, et al. KRAS-MEK signaling controls Ago2 sorting into exosomes. Cell Rep. (2016) 15:978-87. doi: 10.1016/j.celrep.2016.03.085

51. Lu P, Li H, Li N, Singh RN, Bishop CE, Chen X, et al. MEX3C interacts with adaptor-related protein complex 2 and involves in miR-451a exosomal sorting. PLoS ONE. (2017) 12:e0185992. doi: 10.1371/journal.pone.0185992

52. Villarroya-Beltri C, Gutiérrez-Vázquez C, Sánchez-Cabo F, Pérez-Hernández D, Vázquez J, Martin-Cofreces N, et al. Sumoylated hnRNPA2B1 controls the sorting of miRNAs into exosomes through binding to specific motifs. Nat Commun. (2013) 4:2980. doi: 10.1038/ncomms3980

53. Santangelo L, Giurato G, Cicchini C, Montaldo C, Mancone C, Tarallo R, et al. The RNA-binding protein SYNCRIP is a component of the hepatocyte exosomal machinery controlling microRNA sorting. Cell Rep. (2016) 17:799808. doi: 10.1016/j.celrep.2016.09.031

54. Vickers KC, Palmisano BT, Shoucri BM, Shamburek RD, Remaley AT. MicroRNAs are transported in plasma and delivered to recipient cells by high-density lipoproteins. Nat Cell Biol. (2011) 13:423-33. doi: $10.1038 /$ ncb2210

55. Yu B, Zhang X, Li X. Exosomes derived from mesenchymal stem cells. Int $J$ Mol Sci. (2014) 15:4142-57 doi: 10.3390/ijms15034142

56. Chen J, Venkat P, Zacharek A, Chopp M. Neurorestorative therapy for stroke. Front Hum Neurosci. (2014) 8:382. doi: 10.3389/fnhum.2014.00382

57. Alvarez-Erviti L, Seow Y, Yin H, Betts C, Lakhal S, Wood MJ. Delivery of siRNA to the mouse brain by systemic injection of targeted exosomes. Nat Biotechnol. (2011) 29:341-5 doi: 10.1038/nbt.1807

58. Liu S, Chen X, Orcid Id, Bao L, Liu T, Yuan P, et al. Treatment of infarcted heart tissue via the capture and local delivery of circulating exosomes through antibody-conjugated magnetic nanoparticles. Nat Biomed Eng. (2020) 4:1063-75. doi: 10.1038/s41551-020-00637-1

59. Cui Y, Luan J, Li H, Zhou X, Han J. Exosomes derived from mineralizing osteoblasts promote ST2 cell osteogenic differentiation by alteration of microRNA expression. FEBS Lett. (2016) 590:185-92. doi: $10.1002 / 1873-3468.12024$

60. Montecalvo A, Larregina AT, Shufesky WJ, Stolz DB, Sullivan ML, Karlsson JM, et al. Mechanism of transfer of functional microRNAs between mouse dendritic cells via exosomes. Blood. (2012) 119:756-66. doi: 10.1182/blood-2011-02-338004

61. An Y, Orcid Id, Lin S, Tan X, Zhu S, Nie F, et al. Exosomes from adiposederived stem cells and application to skin wound healing. Cell Prolif. (2021) 54:e12993. doi: 10.1111/cpr.12993

62. Betel D, Wilson M, Gabow A, Marks DS, and Sander C. The microRNA.org resource: targets and expression. Nucleic Acids Res. (2008) 36:D149-53. doi: 10.1093/nar/gkm995

63. Yan H, Gao Y, Ding Q, Liu J, Li Y, Jin M, et al. Exosomal micro RNAs derived from dermal papilla cells mediate hair follicle stem cell proliferation and differentiation. Int J Biol Sci. (2019) 15:1368-82. doi: 10.7150/ijbs. 33233

64. Zou XY, Yu Y, Lin S, Zhong L, Sun J, Zhang G, et al. Comprehensive miRNA analysis of human umbilical cord-derived mesenchymal stromal cells and extracellular vesicles. Kidney Blood Press Res. (2018) 43:152-61. doi: $10.1159 / 000487369$

65. Hodjat M, Khan F, Saadat KASM. Epigenetic alterations in aging tooth and the reprogramming potential. Ageing Res Rev. (2020) 63:101140. doi: 10.1016/j.arr.2020.101140

66. Li X, He X, Wang J, Wang D, Cong P, Zhu A, et al. The regulation of exosomederived miRNA on heterogeneity of macrophages in atherosclerotic plaques. Front Immunol. (2020) 11:2175. doi: 10.3389/fimmu.2020.02175

67. Svandova E, Peterkova R, Matalova E, Lesot H. Formation and developmental specification of the odontogenic and osteogenic mesenchymes. Front Cell Dev Biol. (2020) 8:640. doi: $10.3389 /$ fcell.2020.00640
68. Gopal SK, Greening DW, Rai A, Chen M, Xu R, Shafiq A, et al. Extracellular vesicles: their role in cancer biology and epithelial-mesenchymal transition. Biochem J. (2017) 474:21-45. doi: 10.1042/BCJ20160006

69. El Bassit G, Patel RS, Carter G, Shibu V, Patel AA, Song S, et al. MALAT1 in human adipose stem cells modulates survival and alternative splicing of PKC $8 \mathrm{II}$ in HT22 cells. Endocrinology. (2017) 158:183-95 doi: 10.1210/en.2016-1819

70. Zheng Z, Liu L, Zhan Y, Yu S, Kang T. Adipose-derived stem cellderived microvesicle-released miR-210 promoted proliferation, migration and invasion of endothelial cells by regulating RUNX3. Cell Cycle. (2018) 17:1026-33. doi: 10.1080/15384101.2018.1480207

71. Zhuansun Y, Du Y, Huang F, Lin L, Chen R, Jiang S, et al. MSCs exosomal miR-1470 promotes the differentiation of $\mathrm{CD} 4(+) \mathrm{CD} 25(+) \mathrm{FOXP} 3(+)$ Tregs in asthmatic patients by inducing the expression of P27KIP1. Int Immunopharmacol. (2019) 77:105981. doi: 10.1016/j.intimp.2019.105981

72. Xu T, Luo Y, Wang J, Zhang N, Gu C, Li L, et al. Exosomal miRNA-128-3p from mesenchymal stem cells of aged rats regulates. J Nanobiotechnol. (2020) 18:47. doi: 10.1186/s12951-020-00601-w

73. Xu R, Shen X, Si Y, Fu Y, Zhu W, Xiao T, et al. MicroRNA-31a-5p from aging BMSCs links bone formation and resorption in the aged bone marrow microenvironment. Aging Cell. (2018) 17:e12794. doi: 10.1111/acel.12794

74. Liu W, Li L, Rong Y, Qian D, Chen J, Zhou Z, et al. Hypoxic mesenchymal stem cell-derived exosomes promote bone fracture healing by the transfer of miR-126. Acta Biomater. (2020) 103:196-212. doi: 10.1016/j.actbio.2019.12.020

75. Li X, Liu L, Yang J, Yu Y, Chai J, Wang L, et al. Exosome derived from human umbilical cord mesenchymal stem cell mediates MiR-181c attenuating burn-induced excessive inflammation. EBioMedicine. (2016) 8:72-82. doi: 10.1016/j.ebiom.2016.04.030

76. Behera J, Kumar A, Voor MJ, Tyagi N. Exosomal lncRNA-H19 promotes osteogenesis and angiogenesis through mediating Angpt1/Tie2-NO signaling in CBS-heterozygous mice. Theranostics. (2021) 11:7715-34. doi: 10.7150/thno. 58410

77. Liu T, Hu W, Zou X, Xu J, He S, Chang L, et al. Human periodontal ligament stem cell-derived exosomes promote bone regeneration by altering microRNA profiles. Stem Cells Int. (2020) 2020:8852307. doi: $10.1155 / 2020 / 8852307$

78. Park JH, Choi Y, Lim CW, Park JM, Orcid Id, Yu SH, et al. Potential therapeutic effect of micrornas in extracellular vesicles from mesenchymal stem cells against SARS-CoV-2. Cells. (2021) 10:2393. doi: $10.3390 /$ cells 10092393

79. Kang M, Huang CC, Lu Y, Shirazi S, Gajendrareddy P, Ravindran S, et al. Bone regeneration is mediated by macrophage extracellular vesicles. Bone. (2020) 141:115627 doi: 10.1016/j.bone.2020.115627

80. Wozniak AL, Adams A, King KE, Dunn W, Christenson LK, Hung WT, et al. The RNA binding protein FMR1 controls selective exosomal miRNA cargo loading during inflammation. J Cell Biol. (2020) 219:e201912074. doi: $10.1083 /$ jcb. 201912074

81. Fafián-Labora J, Lesende-Rodriguez I, Fernández-Pernas P, SangiaoAlvarellos S, Monserrat L, Arntz OJ, et al. Effect of age on pro-inflammatory miRNAs contained in mesenchymal stem cell-derived extracellular vesicles. Sci Rep. (2017) 7:43923. doi: 10.1038/srep43923

82. Spencer DM, Gauley J, Pisetsky DS. The properties of microparticles from RAW 264.7 macrophage cells undergoing in vitro activation or apoptosis. Innate Immun. (2014) 20:239-48. doi: 10.1177/1753425913492552

83. Umezu T, Tadokoro H, Azuma K, Yoshizawa S, Ohyashiki K, Ohyashiki JH. Exosomal miR-135b shed from hypoxic multiple myeloma cells enhances angiogenesis by targeting factor-inhibiting HIF-1. Blood. (2014) 124:374857. doi: 10.1182/blood-2014-05-576116

84. Lv PY, Gao PF, Tian GJ, Yang YY, Mo FF, Wang ZH, et al. Osteocytederived exosomes induced by mechanical strain promote human periodontal ligament stem cell proliferation and osteogenic differentiation via the miR181b-5p/PTEN/AKT signaling pathway. Stem Cell Res Ther. (2020) 11:295. doi: 10.1186/s13287-020-01815-3

85. Ohno S, Takanashi M, Sudo K, Ueda S, Ishikawa A, Matsuyama $\mathrm{N}$, et al. Systemically injected exosomes targeted to EGFR deliver antitumor microRNA to breast cancer cells. Mol Ther. (2013) 21:185-91. doi: $10.1038 / \mathrm{mt} .2012 .180$ 
86. Qu Y, Zhang Q, Cai X, Li F, Ma Z, Xu M, et al. Exosomes derived from miR-181-5p-modified adipose-derived mesenchymal stem cells prevent liver fibrosis via autophagy activation. J Cell Mol Med. (2017) 21:2491-502. doi: $10.1111 / \mathrm{jcmm} .13170$

87. Momen-Heravi F, Bala S, Bukong T, Szabo G. Exosome-mediated delivery of functionally active miRNA-155 inhibitor to macrophages. Nanomedicine. (2014) 10:1517-27. doi: 10.1016/j.nano.2014.03.014

88. He J, Jiang YL, Wang Y, Tian XJ, Sun SR. Micro-vesicles from mesenchymal stem cells over-expressing miR-34a inhibit transforming growth factor- $\beta 1$ induced epithelial-mesenchymal transition in renal tubular epithelial cells in vitro. Chin Med J. (2020) 133:800-7. doi: 10.1097/CM9.0000000000000720

89. Liu W, Huang J, Chen F, Xie D, Wang L, Ye C, et al. MSC-derived small extracellular vesicles overexpressing miR-20a promoted the osteointegration of porous titanium alloy by enhancing osteogenesis via targeting BAMBI. Stem Cell Res Ther. (2021) 12:348. doi: 10.1186/s13287-021-02303-y

90. Chen S, X. Id- Orcid, Tang Y, Liu Y, Zhang P, Lv L, et al. Exosomes derived from miR-375-overexpressing human adipose mesenchymal stem cells promote bone regeneration. Cell Prolif. (2019) 52:e12669. doi: $10.1111 /$ cpr.12669

91. Hu W, Liu C, Bi ZY, Zhou Q, Zhang H, Li LL, et al. Comprehensive landscape of extracellular vesicle-derived RNAs in cancer initiation, progression, metastasis and cancer immunology. Mol Cancer. (2020) 19:102. doi: 10.1186/s12943-020-01199-1

92. Zhang B, Orcid Id, Yang Y, Xiang L, Orcid Id, Zhao Z, et al. Adipose-derived exosomes: a novel adipokine in obesity-associated diabetes. J Cell Physiol. (2019) 234:16692-702. doi: 10.1002/jcp.28354

93. Fatima F, Ekstrom K, Nazarenko I, Maugeri M, Valadi H, Hill AF, et al. Non-coding RNAs in mesenchymal stem cell-derived extracellular vesicles: deciphering regulatory roles in stem cell potency, inflammatory resolve, and tissue regeneration. Front Genet. (2017) 8:161. doi: 10.3389/fgene.2017.00161

94. Deregibus MC, Tetta C, Camussi G. The dynamic stem cell microenvironment is orchestrated by microvesicle-mediated transfer of genetic information. Histol Histopathol. (2010) 25:397-404. doi: 10.14670/HH-25.397

95. Reis LA, Borges FT, Simões MJ, Borges AA, Sinigaglia-Coimbra R, Schor N. Bone marrow-derived mesenchymal stem cells repaired but did not prevent gentamicin-induced acute kidney injury through paracrine effects in rats. PLoS ONE. (2012) 7:e44092. doi: 10.1371/journal.pone.0044092

96. Whiteside TL. Exosome and mesenchymal stem cell cross-talk in the tumor microenvironment. Semin Immunol. (2018) 35:69-79. doi: 10.1016/j.smim.2017.12.003

97. Liao W, Ning Y, Xu HJ, Zou WZ, Hu J, Liu XZ, et al. BMSC-derived exosomes carrying microRNA-122-5p promote proliferation of osteoblasts in osteonecrosis of the femoral head. Clin Sci. (2019) 133:1955-75 doi: 10.1042/CS20181064

98. Zhuang XM, Zhou B. Exosome secreted by human gingival fibroblasts in radiation therapy inhibits osteogenic differentiation of bone mesenchymal stem cells by transferring miR-23a. Biomed Pharmacother. (2020) 131:110672. doi: 10.1016/j.biopha.2020.110672

99. Léveillé N, Baglio SR. Exosome-transferred lncRNAs at the core of cancer bone lesions. Crit Rev Oncol Hematol. (2019) 139:125-7. doi: 10.1016/j.critrevonc.2019.03.002

100. Jiang LB, Tian L, Zhang CG. Bone marrow stem cells-derived exosomes extracted from osteoporosis patients inhibit osteogenesis via microRNA-21/SMAD7. Eur Rev Med Pharmacol Sci. (2018) 22:6221-9. doi: 10.26355/eurrev_201810_16028

101. Li Y, Wang J, Ma Y, Du W, Feng K, Wang S, et al. miR-101-loaded exosomes secreted by bone marrow mesenchymal stem cells requires the FBXW7/HIF1 $\alpha /$ FOXP3 axis, facilitating osteogenic differentiation. J Cell Physiol. (2021) 236:4258-72. doi: 10.1002/jcp.30027

102. Xie Y, Chen Y, Zhang L, Ge W, Tang P. The roles of bone-derived exosomes and exosomal microRNAs in regulating bone remodelling. J Cell Mol Med. (2017) 21:1033-41. doi: 10.1111/jcmm.13039

103. Zhang D, Li Q, Rao L, Yi B, Xu Q. Effect of 5-Aza-2'-deoxycytidine on odontogenic differentiation of human dental pulp cells. J Endod. (2015) 41:640-5. doi: 10.1016/j.joen.2014.12.006

104. Fu B, Wang H, Wang J, Barouhas I, Liu W, Shuboy A, et al. Epigenetic regulation of BMP2 by 1,25-dihydroxyvitamin D3 through DNA methylation. PLoS ONE. (2013) 8:e61423. doi: 10.1371/journal.pone.00 61423

105. Jiang N, Xiang L, He L, Yang G, Zheng J, Wang C, et al. Exosomes mediate epithelium-mesenchyme crosstalk in organ development. ACS Nano. (2017) 11:7736-46. doi: 10.1021/acsnano.7b01087

106. Mo C, Huang B, Zhuang J, Jiang S, Guo S, Mao X, et al. LncRNA nuclearenriched abundant transcript 1 shuttled by prostate cancer. Clin Transl Med. (2021) 11:e493. doi: 10.1002/ctm2.493

107. Du W, Su L, Zhang N, Wang H. Exosomes derived from preadipocytes improve osteogenic differentiation, potentially via reduced miR-223 expression. Mol Med Rep. (2019) 19:951-8. doi: 10.3892/mmr.2018.9760

108. Zhang Y, Cao X, Li P, Fan Y, Zhang L, Ma X, et al. microRNA-935modified bone marrow mesenchymal stem cells-derived exosomes enhance osteoblast proliferation and differentiation in osteoporotic rats. Life Sci. (2021) 272:119204. doi: 10.1016/j.lfs.2021.119204

109. Mao G, Zhang Z, Hu S, Zhang Z, Chang Z, Huang Z, et al. Exosomes derived from miR-92a-3p-overexpressing human mesenchymal stem cells enhance chondrogenesis and prevent the development of osteoarthritis. Stem Cell Res Ther. (2018) 9:247. doi: 10.1186/s13287-018-1004-0

110. Sun H, Orcid Id, Hu S, Zhang Z, Lun J, Liao W, et al. Expression of exosomal microRNAs during chondrogenic differentiation of human bone mesenchymal stem cells. J Cell Biochem. (2019) 120:171-81. doi: $10.1002 / j$ cb. 27289

111. Li Z, Wang Y, Xiang S, Zheng Z, Bian Y, Feng B, et al. Chondrocytes-derived exosomal miR-8485 regulated the $\mathrm{Wnt} / \beta$-catenin pathways to promote chondrogenic differentiation of BMSCs. Biochem Biophys Res Commun. (2020) 523:506-13. doi: 10.1016/j.bbrc.2019.12.065

112. Mao G, Hu S, Zhang Z, Wu P, Zhao X, Lin R, et al. Exosomal miR-95-5p regulates chondrogenesis and cartilage degradation via histone deacetylase 2/8. J Cell Mol Med. (2018) 22:5354-66. doi: 10.1111/jcmm.13808

113. Zhao C, Chen JY, Peng WM, Yuan B, Bi Q, Xu YJ. Exosomes from adiposederived stem cells promote chondrogenesis and suppress inflammation by upregulating miR-145 and miR-221. Mol Med Rep. (2020) 21:1881-9. doi: $10.3892 / \mathrm{mmr} .2020 .10982$

114. Wu M, Orcid Id, Liu X, Orcid Id, Li Z, Huang X, et al. SHED aggregate exosomes shuttled miR-26a promote angiogenesis in pulp regeneration via TGF- $\beta /$ SMAD2/3 signalling. Cell Prolif. (2021) 54:e13074. doi: 10.1111/cpr.13074

115. Gao L, Mei S, Zhang S, Qin Q, Li H, Liao Y, et al. Cardio-renal exosomes in myocardial infarction serum regulate proangiogenic paracrine signaling in adipose mesenchymal stem cells. Theranostics. (2020) 10:106073. doi: $10.7150 /$ thno. 37678

116. Xin H, Katakowski M, Wang F, Qian JY, Liu XS, Ali MM, et al. MicroRNA cluster miR-17-92 cluster in exosomes enhance neuroplasticity and functional recovery after stroke in rats. Stroke. (2017) 48:747-53. doi: 10.1161/STROKEAHA.116.015204

117. Xin H, Li Y, Buller B, Katakowski M, Zhang Y, Wang X, et al. Exosomemediated transfer of miR-133b from multipotent mesenchymal stromal cells to neural cells contributes to neurite outgrowth. Stem Cells. (2012) 30:1556-64. doi: 10.1002/stem.1129

118. Huang S, Ge X, Yu J, Han Z, Yin Z, Li Y, et al. Increased miR-124-3p in microglial exosomes following traumatic brain injury inhibits neuronal inflammation and contributes to neurite outgrowth via their transfer into neurons. FASEB J. (2018) 32:512-28. doi: 10.1096/fj.201700673r

119. Huang JH, Xu Y, Yin XM, Lin FY. Exosomes derived from miR-126modified MSCs promote angiogenesis and neurogenesis and attenuate apoptosis after spinal cord injury in rats. Neuroscience. (2020) 424:133-45. doi: 10.1016/j.neuroscience.2019.10.043

120. Wang Y, Lai X, Wu D, Liu B, Wang N, Rong L, et al. Umbilical mesenchymal stem cell-derived exosomes facilitate spinal cord functional recovery through the miR-199a-3p/145-5p-mediated NGF/TrkA signaling pathway in rats. Stem Cell Res Ther. (2021) 12:117. doi: 10.1186/s13287-021-02629-7

121. Wan Z, Orcid Id, Chen X, Gao X, Dong Y, Zhao Y, et al. Chronic myeloid leukemia-derived exosomes attenuate adipogenesis of adipose derived mesenchymal stem cells via transporting miR-92a-3p. J Cell Physiol. (2019) 234:21274-83. doi: 10.1002/jcp.28732

122. Wang Y, Chen W, Zhao L, Li Y, Liu Z, Gao H, et al. Obesity regulates miR-467/HoxA10 axis on osteogenic differentiation and fracture healing by 
BMSC-derived exosome LncRNA H19. J Cell Mol Med. (2021) 25:1712-24. doi: $10.1111 / \mathrm{jcmm} .16273$

123. Boilard E. Extracellular vesicles and their content in bioactive lipid mediators: more than a sack of microRNA. J Lipid Res. (2018) 59:2037-2046. doi: 10.1194/jlr.R084640

124. Venkat P, Chopp M, Chen J, Orcid Id. Cell-based and exosome therapy in diabetic stroke. Stem Cells Transl Med. (2018) 7:451-5. doi: $10.1002 / \mathrm{sctm} .18-0014$

125. Haraszti RA, Miller R, Stoppato M, Sere YY, Coles A, Didiot MC, et al. Exosomes produced from 3D cultures of MSCs by tangential flow filtration show higher yield and improved activity. Mol Ther. (2018) 26:2838-47. doi: 10.1016/j.ymthe.2018.09.015

126. Luo ZW, Li FX, Liu YW, Rao SS, Yin H, Huang J, et al. Aptamerfunctionalized exosomes from bone marrow stromal cells target bone to promote bone regeneration. Nanoscale. (2019) 11:20884-92. doi: 10.1039/C9NR02791B

127. Hu X, Zhong Y, Kong Y, Chen Y, Feng J, Zheng J, et al. Lineagespecific exosomes promote the odontogenic differentiation of human dental pulp stem cells (DPSCs) through TGF $\beta 1 /$ smads signaling pathway via transfer of microRNAs. Stem Cell Res Ther. (2019) 10:170. doi: 10.1186/s13287-019-1278-x

128. Galieva LR, James V, Mukhamedshina YO, Rizvanov AA. Therapeutic potential of extracellular vesicles for the treatment of nerve disorders. Front Neurosci. (2019) 13:163. doi: 10.3389/fnins.2019.00163

129. Colao IL, Corteling R, Bracewell D, Wall I. Manufacturing exosomes: a promising therapeutic platform. Trends Mol Med. (2018) 24:242-56. doi: 10.1016/j.molmed.2018.01.006
130. Caporali A, Martello A, Miscianinov V, Maselli D, Vono R, Spinetti G. Contribution of pericyte paracrine regulation of the endothelium to angiogenesis. Pharmacol Ther. (2017) 171:56-64. doi: 10.1016/j.pharmthera.2016. 10.001

131. Zhang D, Lee H, Zhu Z, Minhas JK, Jin Y. Enrichment of selective miRNAs in exosomes and delivery of exosomal miRNAs in vitro and in vivo. Am J Physiol Lung Cell Mol Physiol. (2017) 312:L110-21. doi: 10.1152/ajplung.004 23.2016

Conflict of Interest: The authors declare that the research was conducted in the absence of any commercial or financial relationships that could be construed as a potential conflict of interest.

Publisher's Note: All claims expressed in this article are solely those of the authors and do not necessarily represent those of their affiliated organizations, or those of the publisher, the editors and the reviewers. Any product that may be evaluated in this article, or claim that may be made by its manufacturer, is not guaranteed or endorsed by the publisher.

Copyright (c) 2022 Yan and Yu. This is an open-access article distributed under the terms of the Creative Commons Attribution License (CC BY). The use, distribution or reproduction in other forums is permitted, provided the original author(s) and the copyright owner(s) are credited and that the original publication in this journal is cited, in accordance with accepted academic practice. No use, distribution or reproduction is permitted which does not comply with these terms. 